\title{
Overexpression of activated leukocute cell adhesion molecule in gastric cancer is associated with advanced stages and poor prognosis and miR-9 deregulation
}

\author{
MIN YE ${ }^{1}$, YAN-LEI DU ${ }^{1}$, YU-QIANG NIE ${ }^{1}$, ZHI-WEI ZHOU $^{2}$, JIE CAO $^{3}$ and YING-FEI LI ${ }^{1}$ \\ ${ }^{1}$ Department of Gastroenterology and Hepatology, Guangzhou Key Laboratory of Digestive Disease, \\ Guangzhou First People's Hospital, Guangzhou Medical University, Guangzhou, Guangdong 510180; \\ ${ }^{2}$ Department of Gastroenterology, Luohu District People's Hospital, Shenzhen, Guangdong 518000; \\ ${ }^{3}$ Department of General Surgery, Guangzhou First People's Hospital, Guangzhou Medical University, \\ Guangzhou, Guangdong 510180, P.R. China
}

Received December 19, 2013; Accepted August 5, 2014

DOI: $10.3892 / \mathrm{mmr} .2014 .2933$

\begin{abstract}
Activated leukocyte cell adhesion molecule (ALCAM) has been identified as a novel potential molecular marker of human tumors. The present study aimed to assess ALCAM as a prognostic marker for gastric cancer (GC), and to explore the mRNA deregulation underlying the abnormal expression of ALCAM. The mRNA and protein expression of ALCAM in GC and adjacent non-tumor tissues from 66 patients with GC were analyzed. The association between miR-9 and ALCAM mRNA expression was determined by quantitative polymerase chain reaction. Serum soluble ALCAM (sALCAM) was analyzed by ELISA in 72 patients with GC, 82 patients with gastric precancerous lesions and 73 controls. ALCAM and sALCAM levels were associated with certain clinicopathological variables, including overall survival. Compared with the non-tumor tissues, the expression of ALCAM mRNA in the GC tissues was significantly upregulated $(\mathrm{P}=0.013)$. The expression of miR-9 was reduced and inversely correlated with ALCAM mRNA levels in GC tissues and cell lines. The ALCAM mRNA level was reduced following ectopic overexpression of miR-9 in SGC-7901 human gastric cancer cells. The rates of membranous and cytoplasmic expression of ALCAM in GC tissues were 59.1 and $48.48 \%$, respectively, and the serum sALCAM levels were significantly elevated in patients with GC. Elevated ALCAM mRNA, membranous ALCAM expression in GC tissues and high sALCAM levels are associated with advanced tumor stage,
\end{abstract}

Correspondence to: Dr Yu-Qiang Nie, Department of Gastroenterology and Hepatology, Guangzhou Key laboratory of Digestive Disease, Guangzhou First People's Hospital, Guangzhou Medical University, Guangzhou, No. 1 Panfu Road, Guangdong 510180, P.R. China

E-mail: nieyq@medmail.com.cn

Key words: ALCAM, sALCAM, miR-9, gastric cancer lymphatic invasion and shorter overall survival duration. The results of the current study indicated that membranous ALCAM expression and high serum sALCAM levels are independent prognostic markers of poor survival for patients with GC, and that the overexpression of ALCAM may be due to the downregulation of miR-9.

\section{Introduction}

Activated leukocyte cell adhesion molecule (ALCAM), also known as cluster of differentiation 166 (CD166), is a highly conserved $110 \mathrm{kDa}$ multidomain transmembrane type 1 glycoprotein of the immunoglobulin superfamily (1). ALCAM consists of a NH2-terminal hydrophobic signal peptide, followed by extracellular, transmembrane and cytoplasmic domains (2). ALCAM was identified as a CD6 ligand and is expressed on activated leukocytes, fibroblasts, epithelia and neurons (3). ALCAM is involved in osteogenesis (4), neurite extension (5), hematopoiesis (6) and embryonal implantation in the uterus (7), and mediates homotypic/heterotypic interactions between tumor cells and between endothelial and tumor cells (8). Previously, alterations in the expression of ALCAM mRNA and protein have been reported in several human tumors, including prostate cancer (9), colon cancer (10), breast cancer (11), glioblastoma (12) and non-small-cell lung cancer (13). In metastatic melanoma,ALCAM controls the transition from local cell proliferation to tissue invasion and functions as a cell surface sensor for cell density in metastatic melanoma (14).

The soluble isoform of ALCAM (sALCAM) is an alternative short transcript that comprises only the first 3'-exon. The transcript contains a single Ig domain and lacks a transmembrane domain. : In addition to a regulatory effect on ALCAM function, sALCAM has an ALCAM independent effect in endothelial cell assays (15). sALCAM serum levels have been shown to be elevated in patients with pancreatic (16) and esophageal (17) cancer, and elevated sALCAM levels in peripheral blood are independent prognostic markers of poor survival for patients (17). 
microRNAs (miRNAs) mediate translational repression or direct mRNA cleavage by binding complementary sequences of the mRNA 3' untranslated region (3' UTR) (18). Candidate miRNAs of target mRNAs have been computationally predicted using several bioinformatic tools $(19,20)$. In human hepatoma cell lines, ALCAM was verified as a target gene of miR-9 by luciferase reporter and western blot assays (21). miR-9 is significantly reduced in highly invasive uveal melanoma cell lines, and has been demonstrated to suppress migration and invasion partly through downregulation of the NF- $\kappa$ B1 signaling pathway (22). The anti-proliferative and pro-apoptotic activity of miR-9 via the direct targeting of MTHFD2 may contribute to tumor suppressor-like activity (23).

Gastric cancer (GC) is one of the most common malignancies and a major leading cause of cancer-related mortality worldwide (24). Subsequent to curative surgery and multimodal therapeutic approaches, certain patients with GC undergo an unexpected postoperative course, largely due to a lack of adequate predictive biomarkers and valid therapeutic targets. Ishigami et al (25) suggested that membranous CD166-positivity may be a promising prognostic marker in GC (25). However, ALCAM and sALCAM expression profiles have not previously been investigated in GC. The current study aimed to investigate the expression patterns of ALCAM mRNA and protein in GC tissues and the serum sALCAM levels, and to correlate ALCAM expression with clinicopathological parameters, including survival duration. It was hypothesized that miRNAs are associated with ALCAM overexpression in GC, therefore the functions of computationally predicted miR-9 in GC cells were analyzed.

\section{Materials and methods}

Patients. All blood and tissue samples were obtained from patients who had presented to the Guangzhou First People's hospital (Guangzhou, China) and the study protocol was approved by the Ethics Committee. Written consent was obtained from all participants prior to surgery or blood sample collection.

Primary GC $(n=66)$ and adjacent non-tumor tissues $(>5 \mathrm{~cm}$ away from the margin of the tumor) were snap-frozen in liquid nitrogen immediately subsequent to surgery, and then stored at $-80^{\circ} \mathrm{C}$ until use. The patients were composed of $41(62.12 \%)$ males and $25(37.88 \%)$ females, with ages ranging from 31 to 78 years (median 63 years). None of the patients had received chemotherapy and/or radiotherapy prior to surgery. The tissue samples were formalin (10\%;Shjisbio, Shanghai, China)-fixed and paraffin (Shjisbo)-embedded, then sliced into $4-\mu \mathrm{m}$ sections for immunohistochemistry. For serum sALCAM quantification, peripheral blood samples were obtained from 72 patients with GC (26 females and 46 males, median age 67 years), 82 patients with precancerous lesion of gastric cancer (PLGC; 30 females and 52 males, median age 62 years) and 73 blood-bank donors (28 females and 45 males, median age 64 years) as healthy controls. Exclusion criteria included patients with other malignant tumors and severe diseases in other organs. Lauren's pathohistological classification (26) of GC was used, and PLGC was diagnosed according to the World Health Organization classification system (27).
The histological diagnosis of each case was confirmed by two experienced pathologists independently.

For patients with GC, data were collected on age, gender and tumor histology, and the tumor stage was determined according to the 2010 tumor-node-metastasis (TNM) classification of the Union for International Cancer Control and American Joint Committee on Cancer (27). The clinical follow-up data were obtained from outpatient medical records and phone interviews with patients and their family members. Overall survival (OS) was defined as the duration between the date of diagnosis and the date of mortality or last follow-up appointment. Patients with GC who died of unrelated diseases were regarded as censored cases.

Cell culture. The human GC cell lines MGC-803, BGC-823, HGC-27 and SGC-7901, and the normal human gastric epithelial cell line GES-1 were purchased from Landerbio (Guangzhou, China). HGC-27 cells were cultured in DMEM/F12 complete medium (Hyclone Laboratories, Inc., Logan, UT, USA), while the other cell lines were cultured in RPMI 1640 (Gibco Life Technologies, Grand Island, NY, USA) medium supplemented with $10 \%$ heat-inactivated fetal bovine serum plus $1 \%$ penicillin and streptomycin. The cell lines were kept in a humidified atmosphere with $5 \% \mathrm{CO}_{2}$ at $37^{\circ} \mathrm{C}$.

Transfection. Synthesized miR-9 mimic and negative control (NC) were purchased from Guangzhou RiboBio Co., Ltd. (Guangzhou, China). The cells were transfected with the mimic or the $\mathrm{NC}$ at $50 \mathrm{nM}$, according to the manufacturer's instructions. The cells were transfected using Lipofectamine 2000 (Invitrogen Life Technologies, USA) at 30-50\% confluence, according to the manufacturer's instructions and were harvested following 12, 24 and $48 \mathrm{~h}$ of transfection.

RNA extraction, reverse transcription $(R T)$ and quantitative polymerase chain reaction $(q P C R)$. Total RNA was extracted from the tissues and cell lines using TRIzol ${ }^{\circledR}$ (Ambion Life Technologies, Carlsbad, CA, USA) according to the manufacturer's instructions. Total cDNA was obtained from the RT reaction using PrimeScript RT Reagent Kit (Takara Bio, Inc., Otsu, Japan). ALCAM expression was anlayzed by qPCR using a Stratagene SYBR Green I assay (Agilent Technologies, Inc., Santa Clara, CA, USA) and normalized to that of GAPDH. The sequences of the primers (Invitrogen, Lige Technologies) were as follows: ALCAM F 5'-TTTTACTTACCAGGACAGC-3' and R 5'-GACATAGTTTCCAGCATC-3'; GAPDH F 5'-GCA CCGTCAAGGCTGAGAA C-3' and R 5'-TGGTGAAGAC GCCAGTGGA-3'. The expression levels of miR-9 in the cell lines and tissues were determined using miRACLE cDNA Synthesis Kit and miRACLE qPCR miRNA Master Mix (Genetimes Technology, Inc., Shanghai, China). The miR-9 levels were normalized to the U6 snRNA levels.

Immunohistochemical staining. For 66 pairs of GC and adjacent non-tumor tissues, antigen retrieval was achieved by pressure cooking in citric acid antigen repairing buffer ( $\mathrm{pH}$ 6.0; Shjisbio). The sections were treated with 3\% $\mathrm{H}_{2} \mathrm{O}_{2}$, followed by treatment with bovine serum (Hyclone Laboratores, Inc.,). The primary mouse-derived monoclonal anti-CD166 antibody (Abcam, Cambridge, MA, USA) was 
Table I. Correlations between mRNA expression of activated leukocyte cell adhesion molecule and clinicopathological features of gastric cancer.

\begin{tabular}{|c|c|c|c|c|}
\hline Characteristics & No. of patients & $\begin{array}{l}\text { No. of patients with elevated } \\
\text { expression in cancer }(\%)\end{array}$ & $\begin{array}{l}\text { No. of patients with } \\
\text { expression in cancer }(\%)\end{array}$ & P-value ${ }^{c}$ \\
\hline Gender & & & & 0.300 \\
\hline Male & 41 & $28(42.42)$ & $13(19.70)$ & \\
\hline Female & 25 & $20(30.30)$ & $5(7.58)$ & \\
\hline Age (years) & & & & 0.920 \\
\hline$\leq 60$ & 30 & $22(33.33)$ & $8(12.12)$ & \\
\hline$>60$ & 36 & $26(39.40)$ & $10(15.15)$ & \\
\hline Tumor histology ${ }^{\mathrm{a}}$ & & & & 0.156 \\
\hline Intestinal & 54 & 37 (56.06) & $17(25.76)$ & \\
\hline Diffuse & 12 & $11(16.67)$ & $1(1.51)$ & \\
\hline TNM stage $^{\mathrm{b}}$ & & & & 0.027 \\
\hline $\mathrm{I} / \mathrm{II}$ & 18 & $9(13.64)$ & $9(13.64)$ & \\
\hline III/IV & 48 & 39 (59.08) & $9(13.64)$ & \\
\hline Lymphatic invasion & & & & 0.010 \\
\hline Yes & 49 & $40(60.60)$ & $9(13.64)$ & \\
\hline No & 17 & $8(12.12)$ & $9(13.64)$ & \\
\hline
\end{tabular}

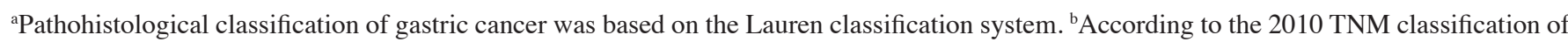
the UICC/AJC. 'Fisher's exact or $\chi^{2}$ test. TNM, tumor node metastasis.

diluted to 1:100 and incubated. The EnVision system (Dako, Glostrup, Denmark) was used to visualize the immunostaining. The tissue sections were independently evaluated by two researchers blinded to the patient characteristics and outcomes.

Sandwich ELISA. For the detection of sALCAM, Costar flexible 96-well microtiter plates (Corning Incorporated, New York, NY, USA) were coated with a monoclonal human anti-mouse ALCAM antibody (\#MAB6561; R\&D Systems, Inc., Minneapolis, MN, USA). Bound protein was detected using a biotinylated polyclonal human anti-goat antibody (\#BAF656; R\&D Systems, Inc.), followed by streptavidin-horseradish peroxidase (\#4800-30-06; R\&D Systems, Inc.) using tetramethylbenzidine as the substrate. The color reaction was stopped, then analyzed at $450 \mathrm{~nm}$ using a Dynatech MR5000 Plate Reader (Dynex Technologies, Vienna, VA, USA). Human ALCAM-Fc protein (R\&D systems, Inc.) was used as an internal standard control. Reproducibility and linearity were examined to ensure that the immunoassay was suitable for measuring clinical serum samples.

Statistical analysis. Group differences were calculated with a t-test or Mann-Whitney test. The Fisher's exact test or $\chi^{2}$ test was used for the comparison of frequencies. The correlation between two factors was evaluated with the Spearman's rank correlation test. Univariate survival analysis was performed using the Kaplan-Meier method and the differences were assessed with the log rank statistic. Independent prognostic factors were estimated by the Cox proportional hazards stepwise regression model. The cut-off level for sALCAM quantification was determined using the Youden index. $\mathrm{P}<0.05$ was considered to indicate a statistically significant difference. All data were analyzed using the SPSS software, version 16.0 (SPSS, Inc., Chicago, IL, USA).

\section{Results}

ALCAM mRNA expression is upregulated in GC, and associated with advanced TNM stage and lymphatic invasion. ALCAM mRNA was upregulated in $72.73 \%$ (48/66) of GC tissues, with an average of a five-fold increase compared with the matched non-tumor (NT) tissues ( $\mathrm{P}=0.013$; Fig. 1A). Elevated ALCAM mRNA in GC tissues was significantly associated with an advanced TNM stage $(\mathrm{P}=0.027)$ and lymphatic invasion $(\mathrm{P}=0.010)$ (Table I). No association was observed with other clinicopathological variables, including gender, age and histology.

ALCAM expression is under the regulation of miR-9. Based on the Sanger miRNA database (http://www.mirbase.org) and TargetScan (http://www.targetscan.org) software, miR-9 was selected, due to a predicted high complementarity between the seed sequence of miR-9 and the two potential binding sites in the 3'-UTR of ALCAM.

Compared with GES-1 cells, ALCAM mRNA expression levels in the gastric cancer cell lines BGC-823, HGC-27 and SGC-7901 were elevated, however, miR-9 levels in these three cell lines were reduced (Fig. 1B). The expression levels of miR-9 were reduced in $62.12 \%(41 / 66)$ of GC tissues compared with the level in NT tissues ( $\mathrm{P}=0.023$; Fig. 1C). The expression levels of miR-9 were inversely correlated with 

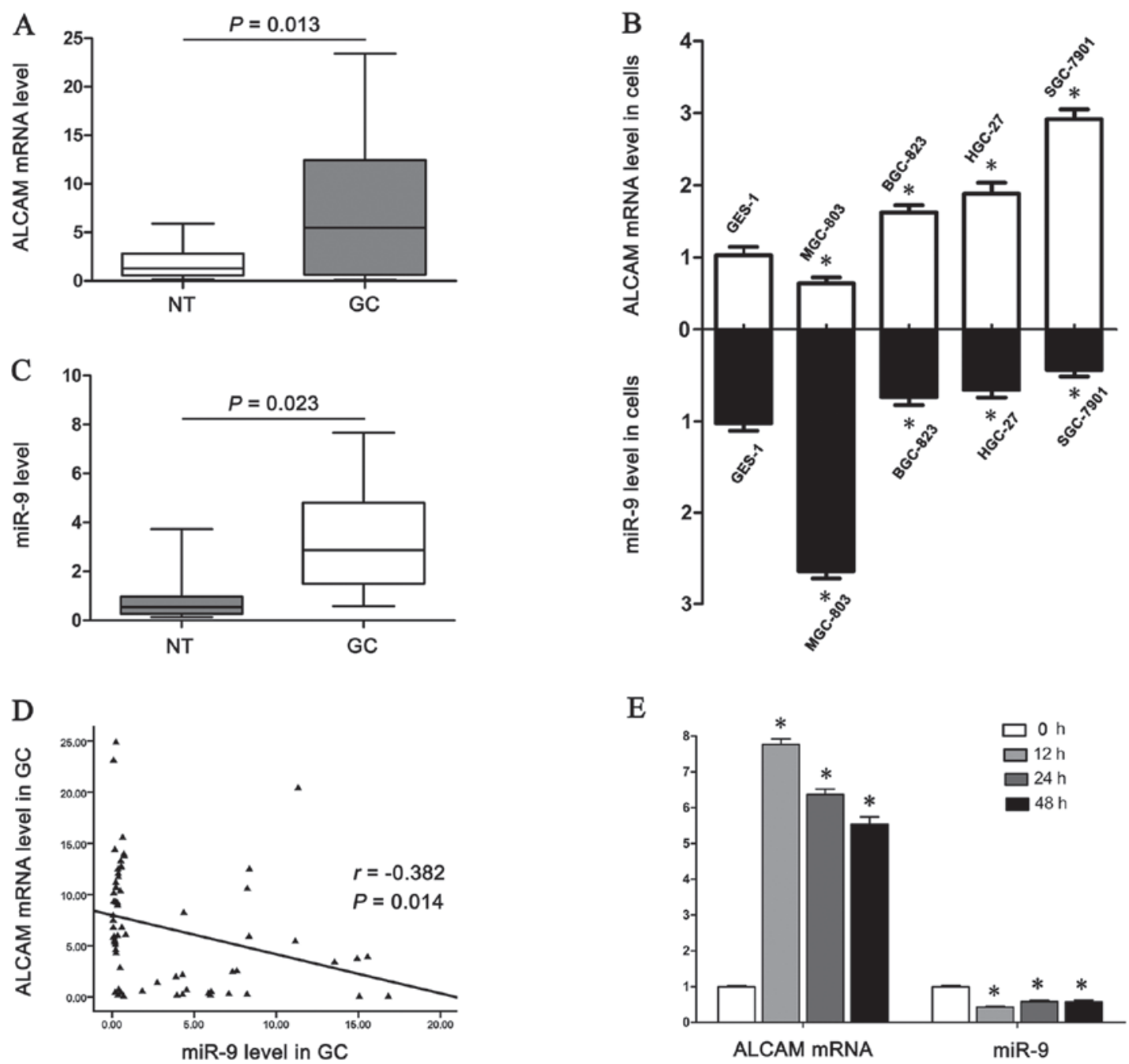

Figure 1. Analysis of ALCAM mRNA and miR-9 expression levels in gastric tissues and cell lines. (A) Comparison of ALCAM mRNA expression levels in 66 paired GC and NT tissues by qPCR. GC tissues (median 5.49) presented significantly higher expression levels than the adjacent NT tissues (median 1.30) ( $\mathrm{P}=0.013$ ). (B) ALCAM mRNA and miR-9 expression levels in four human GC cell lines (MGC-803, BGC-823, HGC-27, SGC-7901) and one normal human gastric epithelium cell line (GES-1). Compared with GES-1, ALCAM mRNA expression levels in the gastric cancer cell lines BGC-823, HGC-27 and SGC-7901 were significantly elevated, whilst the mRNA expression level in MGC-803 cells was significantly reduced. The expression levels of mature miR-9 in BGC-823, HGC-27 and SGC-7901 cell lines were significantly reduced, while the levels significantly increased in MGC- 803 cells ("P<0.05 vs. GES- 1 cells). (C) Comparison of miR-9 expression levels, which were significantly reduced in GC tissues (median 0.55) compared with NT tissues (median 2.87) (P=0.023). (D) Linear regression analysis indicated a significant negative correlation between miR-9 and ALCAM mRNA ( $\mathrm{r}=-0.382, \mathrm{P}=0.014)$. (E) miR-9 and ALCAM mRNA expression level at $0,12,24$ and $48 \mathrm{~h}$ following transfection of miR-9 mimic. The expression of miR-9 was significantly downregulated, while the level of ALCAM mRNA was markedly upregulated, at 12,24 and $48 \mathrm{~h}$ ("P<0.05 vs. 0 h). ALCAM, activated leukocyte cell adhesion molecule; GC, gastric cancer; NT, non-tumor.

those of ALCAM mRNA in GC tissues ( $\mathrm{r}=-0.293, \mathrm{P}=0.014$; Fig. 1D).

miR-9 and ALCAM mRNA levels were quantified following transfection of SGC-7901 cells with the miR-9 mimic and NC. SGC-7901 cells were selected due to having the lowest endogenous miR-9 expression level among the four GC cell lines. At 12, 24 and $48 \mathrm{~h}$ subsequent to transfection of the miR-9 mimic, the expression of miR-9 was significantly decreased, while the level of ALCAM mRNA was markedly increased $(\mathrm{P}<0.05$, Fig. 1E). However, there were no significant differences in the miR-9 or ALCAM mRNA levels in the NC-transfected cells (data not shown).

Clinical significance of ALCAM protein expression and subcellular localization. ALCAM protein expression was identified in the cellular membrane and cytoplasm (Fig. 2). In GC tissues, the positive rates of membranous and cytoplasmic
ALCAM were 59.1\% (39/66) and $48.48 \%$ (32/66), respectively (Table II). In the NT tissues the rates were $27.27 \%$ (18/66) and $22.72 \%(15 / 66)$, respectively. A total of $23 \mathrm{GC}$ tissues were positive for membranous and cytoplasmic ALCAM expression simultaneously.

The upregulated membranous ALCAM expression in cancer tissues was significantly associated with advanced tumor TNM stage $(\mathrm{P}=0.041)$ and lymphatic invasion $(\mathrm{P}=0.040)$; however, the cytoplasmic ALCAM was not associated with any of the clinicopathological variables $(\mathrm{P}>0.05)$ (Table II). The log-rank test indicated that the median OS was significantly reduced in tumors positive for membranous ALCAM (20 months, $n=39$ ) compared with tumors negative for membranous ALCAM (41 months, $n=27)(\mathrm{P}=0.012$; Fig 3A). OS was not significantly associated with cytoplasmic ALCAM in cancer tissues, and the OS median durations in tumors positive for cytoplasmic ALCAM and negative for 
Table II. Association between activated leukocyte cell adhesion molecule subcellular localization in cancer tissues and clinicopathological factors.

\begin{tabular}{|c|c|c|c|c|c|c|c|}
\hline \multirow[b]{2}{*}{ Characteristics } & \multirow[b]{2}{*}{$\begin{array}{l}\text { No. of } \\
\text { patients }\end{array}$} & \multicolumn{3}{|c|}{ Membranous ALCAM in cancer tissues } & \multicolumn{3}{|c|}{ Cytoplasmic ALCAM in cancer tissues } \\
\hline & & $\begin{array}{l}\text { Positive } \\
(\%)\end{array}$ & $\begin{array}{c}\text { Negative } \\
(\%)\end{array}$ & P-value & $\begin{array}{l}\text { Positive } \\
(\%)\end{array}$ & $\begin{array}{c}\text { Negative } \\
(\%)\end{array}$ & P-value \\
\hline Gender & & & & 0.526 & & & 0.569 \\
\hline Male & 41 & $23(34.85)$ & $18(27.27)$ & & $21(31.82)$ & $20(30.30)$ & \\
\hline Female & 25 & $16(24.24)$ & $9(13.64)$ & & $11(16.67)$ & $14(21.21)$ & \\
\hline Age (years) & & & & 0.715 & & & 0.208 \\
\hline$\leq 60$ & 30 & $17(25.76)$ & $13(19.70)$ & & $12(18.18)$ & $18(27.27)$ & \\
\hline$>60$ & 36 & $22(33.33)$ & $14(21.21)$ & & $20(30.30)$ & $16(24.24)$ & \\
\hline Tumor histology & & & & 0.953 & & & 0.450 \\
\hline Intestinal & 54 & $32(48.48)$ & $22(33.33)$ & & $25(37.88)$ & $29(43.94)$ & \\
\hline Diffuse & 12 & $7(10.61)$ & $5(7.58)$ & & $7(10.61)$ & $5(7.58)$ & \\
\hline TNM stage & & & & 0.041 & & & 0.880 \\
\hline $\mathrm{I} / \mathrm{II}$ & 18 & $7(10.61)$ & $11(16.67)$ & & $9(13.64)$ & $9(13.64)$ & \\
\hline III/IV & 48 & $32(48.48)$ & $16(24.24)$ & & $23(34.85)$ & $25(37.88)$ & \\
\hline Lymphatic invasion & & & & 0.040 & & & 0.891 \\
\hline Yes & 49 & $34(51.52)$ & $15(22.73)$ & & $24(36.36)$ & $25(37.88)$ & \\
\hline No & 17 & $5(7.58)$ & $12(18.18)$ & & $8(12.12)$ & $9(13.64)$ & \\
\hline
\end{tabular}

ALCAM, activated leukocyte cell adhesion molecule; TNM, tumor node metastasis.

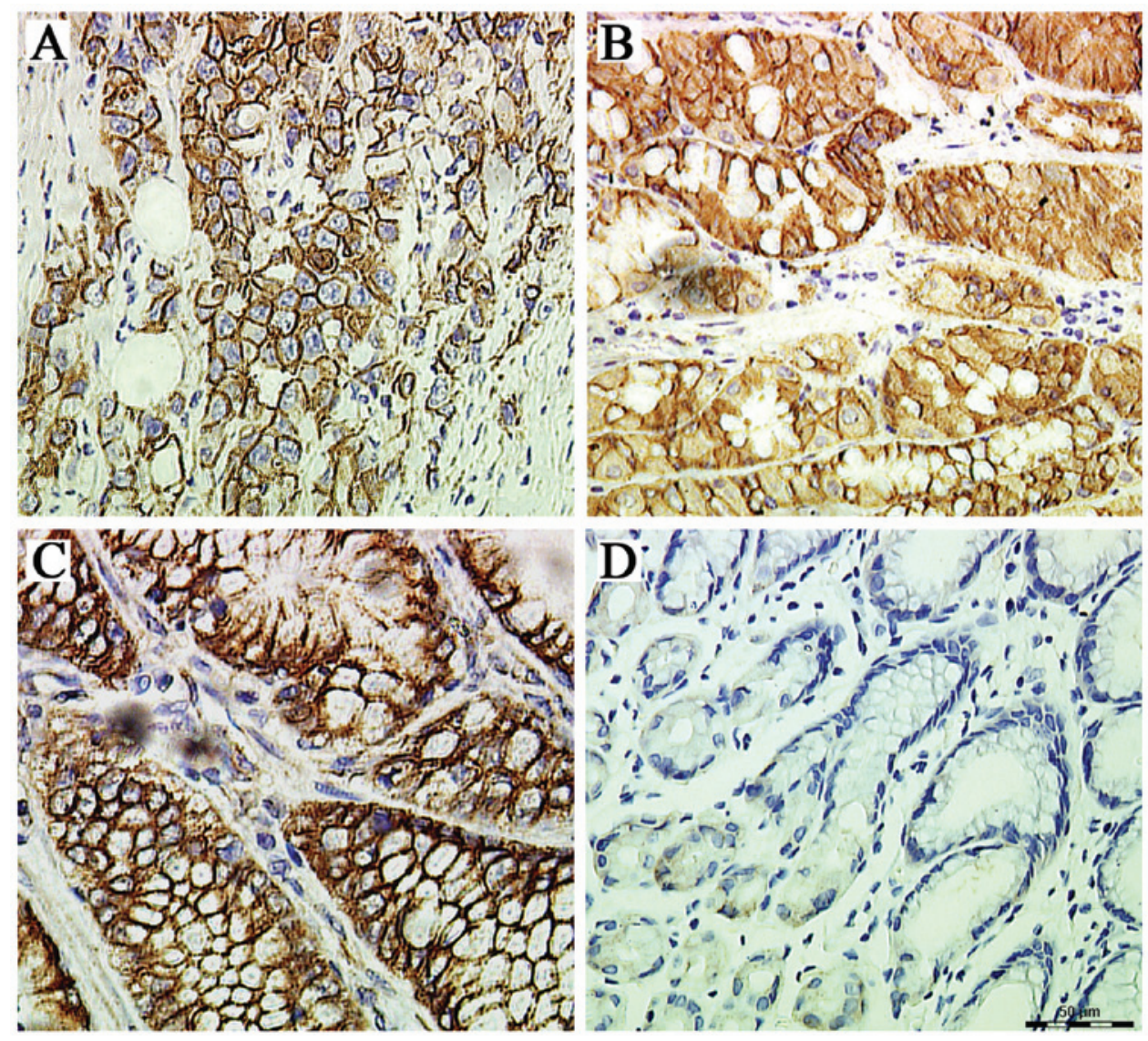

Figure 2. Representative immunohistochemical staining for ALCAM in primary GC and adjacent non-tumor tissues. (A) Strong membranous immunoreactivity for ALCAM in GC tissue. (B) Strong cytoplasmic staining of ALCAM in GC tissue. (C) Strong cytoplasmic and membranous ALCAM expression in GC tissues. (D) Normal gastric mucosa with negative cytoplasmic and membranous staining. (Magnification, x200). ALCAM, activated leukocyte cell adhesion molecule; GC, gastric cancer. 
Table III. Multivariate analysis of factors contributing to overall survival in patients with gastric cancer.

\begin{tabular}{llll}
\hline Characteristics & RR & 95\% CI & P-value $^{\mathrm{a}}$ \\
\hline Gender & 1.280 & $0.702-2.332$ & 0.420 \\
Age & 1.763 & $0.960-3.238$ & 0.068 \\
Tumor histology & 1.289 & $0.876-1.896$ & 0.198 \\
TNM stage & 5.149 & $2.003-13.233$ & 0.001 \\
Lymphatic invasion & 5.562 & $2.164-14.300$ & 0.000 \\
ALCAM membrane expression & 2.251 & $1.170-4.330$ & 0.015 \\
ALCAM cytoplasmic expression & 1.612 & $0.877-2.964$ & 0.124 \\
sALCAM high level & 1.909 & $1.043-3.493$ & 0.036 \\
\hline
\end{tabular}

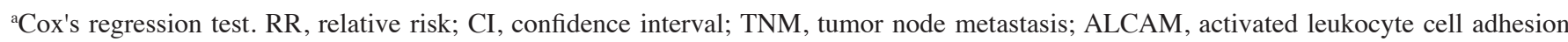
molecule; sALCAM, serum-soluble ALCAM.
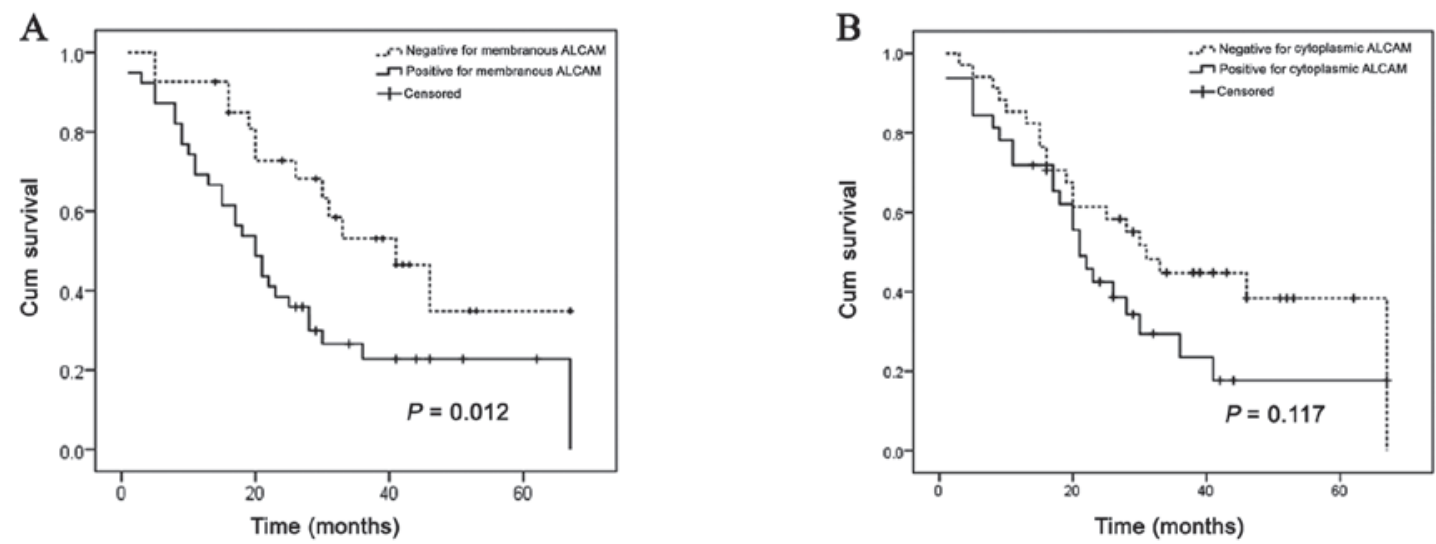

Figure 3. Kaplan-Meier survival curves according to ALCAM subcellular localization of cancer tissues. (A) Overall survival (OS) of patients with gastric cancer, with positive and negative membranous expression of ALCAM. The log-rank test indicated that the median OS was significantly reduced in tumors positive for membranous ALCAM (20 months, $n=39)$ compared with tumors negative for membranous expression of ALCAM (41 months, $n=27)(\mathrm{P}=0.012)$. (B) OS of patients with positive and negative cytoplasmic expression of ALCAM. The median OS durations in tumors positive or negative for cytoplasmic expression of ALCAM were $21(\mathrm{n}=32)$ and 31 months $(\mathrm{n}=34)$, respectively $(\mathrm{P}=0.117)$. ALCAM, activated leukocyte cell adhesion molecule.

cytoplasmic ALCAM were $21(n=32)$ and $31(n=34)$ months, respectively $(\mathrm{P}=0.117$; Fig. $3 \mathrm{~B})$.

Serum sALCAM level and its clinical relevance. No significant difference was observed between the genders or ages of the three groups ( $\mathrm{P}>0.05$; data not shown). The serum level of sALCAM was significantly elevated in GC patients $(50.79 \pm 25.86 \mathrm{ng} / \mathrm{ml})$, compared with PLGC patients $(41.10 \pm 16.30 \mathrm{ng} / \mathrm{ml})$ and healthy blood donors (37.54 $\pm 24.42 \mathrm{ng} / \mathrm{ml})(\mathrm{P}<0.05$; Fig. 4A). The sensitivity-specificity associations for SALCAM to discriminate between GC patients and healthy donors was established using a receiver operating characteristic curve. The optimal cut-off value determined by the Youden index was $44.89 \mathrm{ng} / \mathrm{ml}$, and the area under curve was $0.618(\mathrm{P}=0.008$; Fig. 4B). The specificity of sALCAM $>44.89 \mathrm{ng} / \mathrm{ml}$ in diagnosing GC was $69.33 \%$ with a sensitivity of $41.67 \%$ compared with healthy donors (data not shown). According to the cut-off value, 37 patients with GC were defined as the high-level group, and the other 35 were considered as the low-level group. The Kaplan-Meier survival curve demonstrated a significant difference $(\mathrm{P}=0.031)$ indicating that $\mathrm{OS}$ duration was significantly shorter in the high-level group compared with the low-level group (Fig. 4C).

Regarding the OS, the Cox proportional hazards model (Table III) identified TNM stage $(\mathrm{P}=0.001)$, lymphatic invasion $(\mathrm{P}=0.000)$, membranous ALCAM expression in the primary tumor $(\mathrm{P}=0.015)$ and high serum sALCAM level $(\mathrm{P}=0.036)$ as independent prognostic indicators. Gender, age and tumor histology did not present significance following multivariate analyses $(\mathrm{P}>0.05)$.

\section{Discussion}

In the current study, the results demonstrated that the ALCAM mRNA expression level is elevated in GC tissues and cell lines. In addition, an inverse correlation was observed between miR-9 and ALCAM mRNA expression in the GC tissues and cell lines, and enforced expression of miR-9 led to a reduction of ALCAM mRNA level in the cellular model. Membranous ALCAM was associated with an advanced TNM stage, lymphatic invasion and poor prognosis. The serum sALCAM levels were significantly elevated in GC patients in addition to the membranous ALCAM expression, 

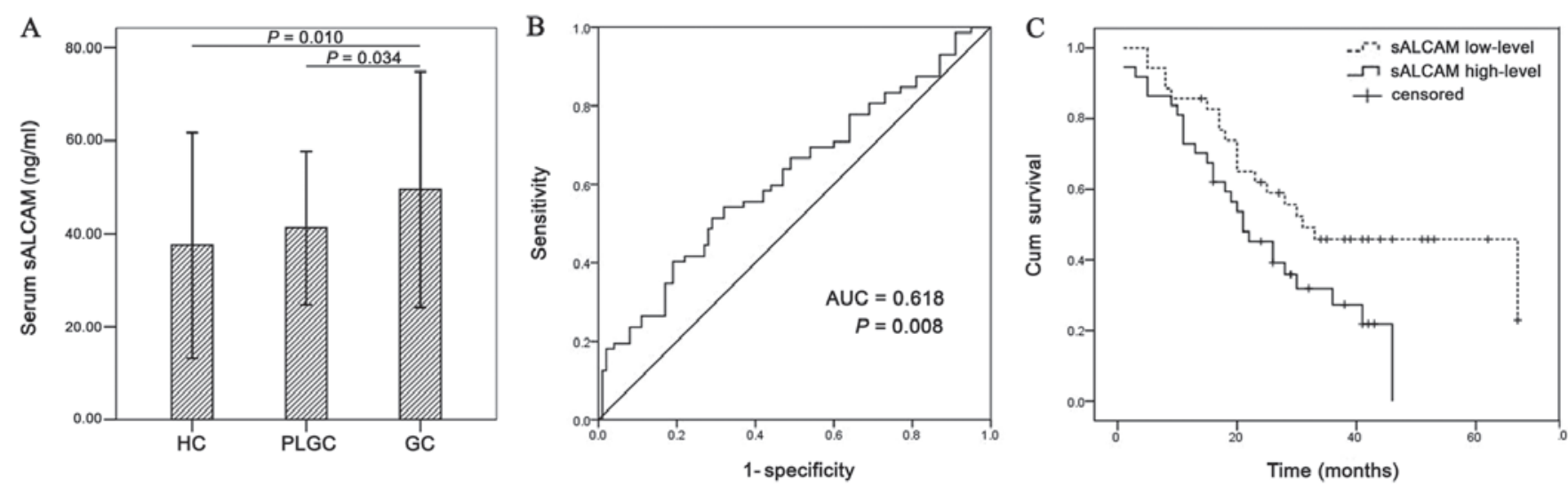

Figure 4. Analysis of serum soluble (s) ALCAM. (A) Analysis of sALCAM levels in patients with gastric cancer (GC), precancerous lesions of gastric cancer (PLGC) and healthy controls (HC). The level of sALCAM was significantly elevated in patients with GC (50.79 $\pm 25.86 \mathrm{ng} / \mathrm{ml})$, compared with patients with PLGC (41.10 $\pm 16.30 \mathrm{ng} / \mathrm{ml})(\mathrm{P}=0.034)$ and $\mathrm{HC}(37.54 \pm 24.42 \mathrm{ng} / \mathrm{ml})(\mathrm{P}=0.010)$. (B) Receiver operating characteristic curve of sALCAM to discriminate between patients with GC and HC. The optimal cut-off value, determined by the Youden index, was $44.89 \mathrm{ng} / \mathrm{ml}$, and the AUC was 0.618 ( $\mathrm{P}=0.008$ ). (C) The overall survival of patients with GC patients with high and low sALCAM levels. The overall survival was significantly shorter in the high-level group (21 months, $\mathrm{n}=37$ ) compared with the low-level group (31 months, $\mathrm{n}=35)(\mathrm{P}=0.031)$. sALCAM, soluble activated leukocyte cell adhesion molecule; AUC, area under curve.

and sALCAM was identified as an independent prognostic indicator.

The association of ALCAM with disease progression in human malignancies was first suggested in malignant melanoma (28). To the best of our knowledge, the current study is the first to present data with respect to ALCAM expression in gastric cancer. The results demostrated that ALCAM levels were significantly increased in cancer tissues with advanced TNM stages and lymphatic invasion. The presence of membranous ALCAM may serve as an indicator of poor prognosis in GC. Consistent with these results, elevated expression levels of ALCAM have previously been detected in all 20 malignant mesothelioma (MM) cell lines, and overexpression of ALCAM was demonstrated to contribute to tumor progression in MM (29). Ishigami et al (25) reported the rates of membranous and cytoplasmic ALCAM expression as 25.4 and $34.4 \%$, respectively, which are lower than the present findings. This may be due to the inclusion of a greater number of patients with an advanced TNM stage (72.72 vs. $24.64 \%)$ in the present cohort, or the innate heterogeneity of GC pathogenesis.

ALCAM is bimodular, and the two modules are required for stable cell adhesion and aggregation (30). ALCAM clustering is essential to obtain stable adhesion and, at the cell surface, actin cytoskeleton-dependent clustering of ALCAM molecules regulate ALCAM mediated cell adhesion (31). In metastatic melanoma BLM cells, ALCAM acts as a cell density sensor and initiates a signal to induce MMP-2 activation (14). According to the type of cadherin adhesion complexes and the cadherin status of the tumor cells, ALCAM may differentially enhance or reduce invasiveness (32). Membranous and cytoplasmic ALCAM expression patterns were identified in GC tissues, as reported in other types of malignancies; however, the biological relevance of these patterns of ALCAM distribution is largely unclear. With respect to subcellular localization, Tomita et al (33) hypothesized that membranous ALCAM may be an activated form that can interact with extracellular components, as it consists of five extracellular domains, which fulfill its original function in homophilic interactions (33). By contrast, loss of ALCAM membrane expression has been implicated as an independent factor of unfavorable prognosis in epithelial ovarian cancer patients (34). The above evidence indicates that the evaluation of the prognostic impact of ALCAM expression should be considered for each type of tumor, based on the subcellular localization and expression levels of ALCAM. The molecular mechanisms by which ALCAM participates in tumor proliferation, motility and metastatic invasion require further investigation.

The extracellular domain of ALCAM (sALCAM) is shed by metalloproteases and functions as an active messenger interacting with surrounding tissues (35). It has been described as a promoter of endothelial cell migration and an inhibitor of endothelial tube formation. sALCAM modulates endothelial cell function through ALCAM-dependent and -independent pathways, and its expression is differentially regulated upon inflammatory stimulation (15). Several studies have reported its potential as a biomarker for different tumor entities. Concurrent with the present study, patients with elevated sALCAM level were identified in a previous study to have significantly worse OS durations, thus it may serve as a potential diagnostic and prognostic serum marker for esophageal cancer (17). The sensitivity and specificity of sALCAM was clearly inferior to the tumor marker most frequently used for pancreatic cancer, CA19-9 (16).

No significant association was identified between the elevated tissue expression and serum level in the patients. The tissue levels of ALCAM may not have correlated with the serum values for several reasons. For example, TNF- $\alpha$ has been demonstrated to induce moderate and persistent upregulation of sALCAM expression in HMVECs, although it exhibits minimal early effects (15). The expression of ALCAM protein does not necessarily result in increased cleavage of sALCAM by proteases such as ADAM17 (36). The flushing of sALCAM into the vascular system may be a consequence of the disruption of anatomical barriers between tumor cells and the bloodstream. In view of these studies $(32,37)$, it is clear that the mechanisms regulating the 
shedding of ALCAM, its dissemination into the surrounding tissue and its entry into the blood system are not clearly understood.

The exact mechanisms involved in the regulation of ALCAM have yet to be elucidated, particularly the mechanism underlying the aberrant expression of ALCAM during malignant transformation. In a study by Wang et al (21), ALCAM mRNA and protein expression levels were upregulated following serum deprivation (SD) in HepG2 and GQY-7701 cells, partly due to the SD-mediated NF- $\kappa \mathrm{B}$ P50/P65 increase, which enhances open chromatin accessibility around the $\kappa \mathrm{B}$ motif independent of SWI/SNF complexes. miRNAs bind the 3'-UTR of target mRNAs and direct mRNA cleavage (38). miR-9 was selected as a candidate in the current study, as two potential binding sites in the 3'-UTR of ALCAM were identified by computational prediction, and there is phylogenic conservation of the binding site sequence among mammals. In human hepatoma cell lines, it was verified that miR-9 targets ALCAM, as ALCAM protein levels were suppressed by miR-9. Following miR-9 mimic transfection, the level of ALCAM mRNA was significantly reduced, indicating that miR-9 is able to downregulate the ALCAM mRNA expression level in GC. Further studies are required to investigate whether miR-9 translationally represses ALCAM protein expression in GC cell lines.

\section{Acknowledgements}

The present study was supported by the Science and Technology Development Plan Project of Guangzhou (no. 2011J4100025), the Key Project of Guangzhou Health Bureau (no. 20121A021004) and the Natural Science Foundation of Guangdong (no. 101510060010 00016), China.

\section{References}

1. Ofori-Acquah SF and King JA: Activated leukocyte cell adhesion molecule: a new paradox in cancer. Transl Res 151: 122-128, 2008.

2. Bowen MA, Patel DD, Li X, et al: Cloning, mapping, and characterization of activated leukocyte-cell adhesion molecule (ALCAM), a CD6 ligand. J Exp Med 181: 2213-2220, 1995.

3. Patel DD, Wee SF, Whichard LP, et al: Identification and characterization of a $100-\mathrm{kD}$ ligand for CD6 on human thymic epithelial cells. J Exp Med 181: 1563-1568, 1995.

4. Bruder SP, Ricalton NS, Boynton RE, et al: Mesenchymal stem cell surface antigen SB-10 corresponds to activated leukocyte cell adhesion molecule and is involved in osteogenic differentiation. J Bone Miner Res 13: 655-663, 1998.

5. Sekine-Aizawa Y, Omori A and Fujita SC: MuSC, a novel member of the immunoglobulin superfamily, is expressed in neurons of a subset of cranial sensory ganglia in the mouse embryo. Eur J Neurosci 10: 2810-2824, 1998.

6. Cortés F, Deschaseaux F, Uchida N, et al: HCA, an immunoglobulin-like adhesion molecule present on the earliest human hematopoietic precursor cells, is also expressed by stromal cells in blood-forming tissues. Blood 93: 826-837, 1999.

7. Fujiwara H, Tatsumi K, Kosaka K, et al: Human blastocysts and endometrial epithelial cells express activated leukocyte cell adhesion molecule (ALCAM/CD166). J Clin Endocrinol Metab 88: 3437-3443, 2003.

8. Weidle UH, Eggle D, Klostermann S and Swart GW: ALCAM/CD166: cancer-related issues. Cancer Genomics Proteomics 7: 231-243, 2010.

9. Kristiansen G, Pilarsky C, Wissmann C, et al: ALCAM/CD166 is up-regulated in low-grade prostate cancer and progressively lost in high-grade lesions. Prostate 54: 34-43, 2003.
10. Weichert W, Knösel T, Bellach J, Dietel M and Kristiansen G: ALCAM/CD166 is overexpressed in colorectal carcinoma and correlates with shortened patient survival. J Clin Pathol 57: 1160-1164, 2004

11. Hein S, Müller V, Köhler N, et al: Biologic role of activated leukocyte cell adhesion molecule overexpression in breast cancer cell lines and clinical tumor tissue. Breast Cancer Res Treat 129: 347-360, 2011.

12. Kijima N, Hosen N, Kagawa N, et al: CD166/activated leukocyte cell adhesion molecule is expressed on glioblastoma progenitor cells and involved in the regulation of tumor cell invasion. Neuro Oncol 14: 1254-1264, 2012.

13. Ishiguro F, Murakami H, Mizuno T, et al: Membranous expression of activated leukocyte cell adhesion molecule contributes to poor prognosis and malignant phenotypes of non-small-cell lung cancer. J Surg Res 179: 24-32, 2013.

14. Lunter PC, van Kilsdonk JW, van Beek H, et al: Activated leukocyte cell adhesion molecule (ALCAM/CD166/MEMD), a novel actor in invasive growth, controls matrix metalloproteinase activity. Cancer Res 65: 8801-8808, 2005.

15. Ikeda K and Quertermous T: Molecular isolation and characterization of a soluble isoform of activated leukocyte cell adhesion molecule that modulates endothelial cell function. J Biol Chem 279: 55315-55323, 2004

16. Tachezy M, Zander H, Marx AH, et al: ALCAM (CD166) expression and serum levels in pancreatic cancer. PLoS One 7: e39018, 2012

17. Tachezy M, Effenberger K, Zander H, et al: ALCAM (CD166) expression and serum levels are markers for poor survival of esophageal cancer patients. Int J Cancer 131: 396-405, 2012.

18. Ambros V: The functions of animal microRNAs. Nature 431: 350-355, 2004.

19. Sethupathy P, Megraw M and Hatzigeorgiou AG: A guide through present computational approaches for the identification of mammalian microRNA targets. Nat Methods 3: 881-886, 2006.

20. Mendes ND, Freitas AT and Sagot MF: Current tools for the identification of miRNA genes and their targets. Nucleic Acids Res 37: 2419-2433, 2009.

21. Wang J, Gu Z, Ni P, et al: NF-kappaB P50/P65 hetero-dimer mediates differential regulation of CD166/ALCAM expression via interaction with micoRNA-9 after serum deprivation, providing evidence for a novel negative auto-regulatory loop. Nucleic Acids Res 39: 6440-6455, 2011.

22. Liu N, Sun Q, Chen J, et al: MicroRNA-9 suppresses uveal melanoma cell migration and invasion through the NF- $\kappa$ B1 pathway. Oncol Rep 28: 961-968, 2012.

23. Selcuklu SD, Donoghue MT, Rehmet K, et al: MicroRNA-9 inhibition of cell proliferation and identification of novel miR-9 targets by transcriptome profiling in breast cancer cells. J Biol Chem 287: 29516-29528, 2012.

24. de Martel C, Forman D and Plummer M: Gastric cancer: epidemiology and risk factors. Gastroenterol Clin North Am 42: 219-240, 2013.

25. Ishigami S, Ueno S, Arigami T, et al: Clinical implication of CD166 expression in gastric cancer. J Surg Oncol 103: 57-61, 2011.

26. Lauren P: The two histological main types of gastric carcinoma: Diffuse and so-called intestinal-type carcinoma. An attempt at a histo-clinical classification.Acta Pathol Microbiol Scand 64: 31-49, 1965

27. Lauwers GY, Carneiro F and Graham DY: Gastric carcinoma. In: WHO Classification of tumors of the digestive system Bosman FT, Carneiro F and Hruban RH (eds). IARC Press, Lyon, pp48-58, 2010.

28. C, van den Oord JJ, van Muijen GN, Weidle UH, Bloemers HP and Swart GW: Activated leukocyte cell adhesion molecule/CD166, a marker of tumor progression in primary malignant melanoma of the skin. Am J Pathol 156: 769-774, 2000.

29. Ishiguro F, Murakami H, Mizuno T, et al: Activated leukocyte cell-adhesion molecule (ALCAM) promotes malignant phenotypes of malignant mesothelioma. J Thorac Oncol 7: 890-899, 2012.

30. van Kempen LC, Nelissen JM, Degen WG, et al: Molecular basis for the homophilic activated leukocyte cell adhesion molecule (ALCAM)-ALCAM interaction. J Biol Chem 276: 25783-25790, 2001.

31. Nelissen JM, Peters IM, de Grooth BG, van Kooyk Y and Figdor CG: Dynamic regulation of activated leukocyte cell adhesion molecule-mediated homotypic cell adhesion through the actin cytoskeleton. Mol Biol Cell 11: 2057-2068, 2000. 
32. Jannie KM, Stipp CS and Weiner JA: ALCAM regulates motility, invasiveness, and adherens junction formation in uveal melanoma cells. PLoS One 7: e39330, 2012.

33. Tomita K, van Bokhoven A, Jansen CF, Bussemakers MJ and Schalken JA: Coordinate recruitment of E-cadherin and ALCAM to cell-cell contacts by alpha-catenin. Biochem Biophys Res Commun 267: 870-874, 2000.

34. Mezzanzanica D, Fabbi M, Bagnoli M, et al: Subcellular localization of activated leukocyte cell adhesion molecule is a molecular predictor of survival in ovarian carcinoma patients. Clin Cancer Res 14: 1726-1733, 2008.

35. Rosso O, Piazza T, Bongarzone I, et al: The ALCAM shedding by the metalloprotease ADAM17/TACE is involved in motility of ovarian carcinoma cells. Mol Cancer Res 5: 1246-1253, 2007.
36. Weidle UH, Eggle D and Klostermann S: L1-CAM as a target for treatment of cancer with monoclonal antibodies. Anticancer Res 29: 4919-4931, 2009 .

37. Kahlert C, Weber H, Mogler C, et al: Increased expression of ALCAM/CD166 in pancreatic cancer is an independent prognostic marker for poor survival and early tumour relapse. $\mathrm{Br}$ J Cancer 101: 457-464, 2009.

38. Bartel DP: MicroRNAs: genomics, biogenesis, mechanism, and function. Cell 116: 281-297, 2004. 